\title{
EXISTENCIA DE SOLUCIONES LOCALES DÉBILES DE UN SISTEMA ACOPLADO DE KIRCHHOFF
}

\author{
Luis Guillermo Huamanlazo Ricci ${ }^{1}$
}

(Recibido: 12/12/2014 - Aceptado: 04/12/2015)

Resumen: En el presente trabajo, estudiamos la existencia de soluciones locales débiles de un sistema acoplado de Kirchhoff. Este sistema se trata de una generalización de sistemas acoplados del tipo Klein - Gordon.

Palabras clave: Solución local, sistema acoplado, Método de Galerkin, método de estimativas de Tartar.

\section{WEAK LOCAL EXISTENCE OF SOLUTIONS OF A COUPLED KIRCHHOFF}

Abstract: In present work, we study weak local existence of solutions of a coupled Kirchhoff. This system is a generalization of coupled Gordo Klein.

Keywords: Local solution, coupled system, Galerkin method, Tartar estimates method.

\section{Introducción}

El objetivo fundamental de este trabajo es estudiar el sistema acoplado siguiente:

$$
(*)\left\{\begin{array}{c}
\left.u_{t t}(x, t)-M\left(\left\|u(t)^{2}\right\|\right) \Delta u(x, t)=v^{2}(x, t) u(x, t) ;(x, t) \in \Omega \times\right] 0, T[ \\
\left.v_{t t}(x, t)-M\left(\|v(t)\|^{2}\right) \Delta v(x, t)=u^{2}(x, t) v(x, t) ;(x, t) \in \Omega \times\right] 0, T[ \\
u(x, 0)=u_{0}(x) ; v(x, 0)=v_{0}(x) ; x \in \Omega \\
u_{t}(x, 0)=u_{1}(x) ; v_{t}(x, 0)=v_{1}(x) ; x \in \Omega \\
\left.u(x, t)=v(x, t)=0 ;(x, t) \in \sum=\partial \Omega \times\right] 0, T[
\end{array}\right.
$$

Donde $\Omega$ es un abierto acotado de $\mathbb{R}^{n}$ con frontera bien regular, $M$ es una función de clase $C^{1}\left(\left[0,+\infty[)\right.\right.$ la cual satisface que $M(S) \geq m_{0}>0$, donde $m_{0}$ es una constante real. Aquí $T>0$ es un número real que se ha escogido arbitrariamente y luego ya se considera fijado. Con el símbolo $\Delta$ representamos el operador Laplaciano definido por $\Delta=\sum_{i=1}^{2} \frac{\partial^{2}}{\partial x_{i}^{2}}$ y con $\|u(t)\|^{2}$ denotamos la norma del espacio de Hilbert $H_{0}^{1}(\Omega)$ definida por

$$
\|u(t)\|^{2}=\int_{\Omega}|\nabla u(x, t)|^{2} d x
$$

El sistema $(*)$ es una generalización de la ecuación

$$
u_{t t}-M\left(\|u(t)\|^{2}\right) \Delta u=|u|^{2} u
$$

Pues en $(*)$ haciendo $u=v$ obtenemos la ecuación anterior.

\footnotetext{
${ }^{1}$ UNMSM, Facultad de Ciencias Matemáticas, e-mail: e-mail: lhrguille@gmail.com
} 


\section{Resultados importantes}

Sea $\Omega$ un abierto acotado de $\mathbb{R}^{2}$, Con frontera bien regular, $T>0$ un número real y $Q$ el cilindro $Q=\Omega \times] 0, T\left[\right.$. Por $(\cdot, \cdot)$ y $|\square|$ denotamos el producto interno y la norma de $L^{2}(\Omega)$ y por $\delta(u, v)$ y $\|\square\|$ denotamos el producto interno y la norma en $H_{0}^{1}(\Omega)$. Recordemos que $a(u, v)$ es la llamada forma de Dirichlet definida por

$$
a(u, v)=\int_{\Omega} \nabla u \nabla v d x
$$

Que también se denota por $((\cdot, \cdot))$ y $\|\square\|$ denotaran el producto interno y la norma de $H_{0}^{1}(\Omega)$. Además $H_{0}^{1}(\Omega)$ es la clausura en $H^{1}(\Omega)$ del espacio $\mathcal{D}(\Omega)$, donde $\mathcal{D}(\Omega)$ denota el espacio de funciones infinitamente diferenciables con soporte contenido en $\Omega$.

Sea X un espacio de Banach y $1 \leq p<\infty$ un número real. Representamos por $L^{p}(0, T ; X)$ al espacio de Banach de funciones con valores vectoriales $u:] 0, T[\rightarrow X$ las cuales son medibles y $\|u(t)\|_{X} \in L^{P}(0, T)$, con la norma

$$
\|u(t)\|_{L^{P}(0, T ; X)}=\left(\int_{0}^{T}\|u(t)\|_{X}^{p} d t\right)^{1 / p}
$$

Por $L^{\infty}(0, T ; X)$ representamos al espacio de Banach de las funciones $\left.u:\right] 0, T[\rightarrow X$ las cuales son medibles y esencialmente acotadas en $\Omega$, con la norma

$$
\|u(t)\|_{L^{\infty}(0, T ; X)}=\operatorname{Sup}_{t \in] 0, T[} \operatorname{ess}|u(t)|_{X}
$$

Además $\mathcal{D}^{\prime}(Q)$ y $\mathcal{D}(0, T)$ denotaran el espacio de distribuciones sobre $Q$ y $] 0, T[$ respectivamente. Todas las funciones escalares consideradas en este trabajo son de valores reales.

En nuestro resultado principal usaremos el siguiente Lema

Lema 2.1 Si $u, v \in H_{0}^{1}(\Omega) \cap H^{2}(\Omega)$ entonces $\exists c>0$ tal que $\left|v^{2} u\right| \leq c\|v\|^{2}\|u\|\left(\Omega \subset \mathbb{R}^{n} ; n=1,2\right)$.

Demostración. Ver Milla Miranda - Medeiros [6]

Teorema 2.2 (Alaoglu-Bourbaki) Sea $X$ un espacio de Banach. Si $\left(u_{m}\right)_{n \in \mathbb{N}}$ satisface que $\left\|u_{m}\right\|_{X} \leq C$. Entonces existe $\left(u_{n_{k}}\right) \subset\left(u_{m}\right), u \in X$ tal que $u_{n_{k}} \stackrel{*}{\rightarrow} u$ débil ${ }^{*}$ en $X$.

Demostración. Ver Brezis, H. [1].

\section{Teorema de la existencia local}

Sean $u_{0}, v_{0} \in H_{0}^{1}(\Omega) \cap H^{2}(\Omega)$ y $u_{1}, v_{1} \in H_{0}^{1}(\Omega)$ entonces existe $T_{0}>0$ con $T_{0}<T$ y funciones $u, v:] 0, T_{0}\left[\rightarrow L^{2}(\Omega)\right.$ tal que

$$
\begin{gathered}
u, v \in L^{\infty}\left(0, T_{0} ; H_{0}^{1}(\Omega) \cap H^{2}(\Omega)\right) \\
u_{t}, v_{t} \in L^{\infty}\left(0, T_{0} ; H_{0}^{1}(\Omega)\right) \\
u_{t t}, v_{t t} \in L^{\infty}\left(0, T_{0} ; L^{2}(\Omega)\right)
\end{gathered}
$$

y

$$
\left.\begin{array}{l}
u_{t t}-M\left(\|u(t)\|^{2}\right) \Delta u=v^{2} u \\
v_{t t}-M\left(\|v(t)\|^{2}\right) \Delta v=u^{2} v
\end{array}\right\} \text { en el sentido débil en } Q
$$

Además

$$
\begin{aligned}
u(0) & =u_{0}, v(0)=v_{0} \\
u_{t}(0) & =u_{1}, v_{t}(0)=v_{1}
\end{aligned}
$$


Demostración. La demostración se hará usando el método de Faedo Galerkin, con argumentos de compacidad combinados con el método de estimativas de Tartar [9]. Este método consiste en etapas.

$\underline{1^{\mathrm{a}} \text { Etapa: Soluciones Aproximadas }}$

Sea $\left(\omega_{\gamma}\right)_{\gamma} \in \mathbb{N}$ una base de $H_{0}^{1}(\Omega)$ formado por las funciones propias del problema de Dirichlet:

$$
\mid \begin{gathered}
\Delta \omega_{j}=\lambda_{j} \omega_{j} \text { en } \Omega \\
\omega_{j}=\text { en } \partial \Omega
\end{gathered}
$$

y sea $V_{m}=\left[\omega_{1}, \omega_{2}, \ldots, \omega_{m}\right]$ el subespacio generado por los primeros $m$ vectores $\omega_{1}, \omega_{2}, \ldots, \omega_{m}$. Continuando con la demostración del teorema. Sean

$$
u_{m}(t)=\sum_{j=1}^{m} g_{j m}(t) \omega_{j} \quad, \quad v_{m}(t)=\sum_{j=1}^{m} h_{j m}(t) \omega_{j}
$$

Soluciones aproximadas en $V_{m}$ donde $g_{j m}, h_{j m} \in C^{2}\left(\left[0, T_{0}\right]\right)$, del problema $(*)$.

\section{Notemos:}

De que $u_{m}(t)$ y $v_{m}(t) \in V_{m}$ es evidente pues son combinaciones lineales finitas de las $m$ funciones $\omega_{j} \in V_{m}\left(g_{j m}(t), h_{j m}(t) \in \mathbb{R}\right)$.

Ellas $\left(u_{m}(t)\right.$ y $\left.v_{m}(t)\right)$ son definidas como soluciones del sistema (S.A):

$$
\begin{array}{r}
\left(u_{m}^{\prime \prime}(t), \omega_{j}\right)+M\left(\left\|u_{m}(t)\right\|^{2}\right) a\left(u_{m}(t), \omega_{j}\right)=\left(v_{m}^{2}(t) u_{m}(t), \omega_{j}\right) \\
\left(v_{m}^{\prime \prime}(t), \omega_{j}\right)+M\left(\left\|v_{m}(t)\right\|^{2}\right) a\left(v_{m}(t), \omega_{j}\right)=\left(u_{m}^{2}(t) v_{m}(t), \omega_{j}\right) ; j=1,2, \ldots, m \\
u_{0}(0)=u_{0 m} ; u_{0 m} \rightarrow u_{0} \text { fuerte en } H_{0}^{1}(\Omega) \cap H^{2}(\Omega) \\
v_{0}(0)=v_{0 m} ; v_{0 m} \rightarrow v_{0} \text { fuerte en } H_{0}^{1}(\Omega) \cap H^{2}(\Omega) \\
u_{m}^{\prime}(0)=u_{1 m} ; u_{1 m} \rightarrow u_{1} \text { fuerte en } H_{0}^{1}(\Omega) \\
v_{m}^{\prime}(0)=v_{1 m} ; v_{1 m} \rightarrow v_{1} \text { fuerte en } H_{0}^{1}(\Omega)
\end{array}
$$

El sistema aproximado (1)-(6) es un sistema de ecuaciones diferenciales ordinarias, el cual por el teorema de Caratheodory nos garantiza que $u_{m}(t)$ y $v_{m}(t)$ son definidas en $\left[0, t_{m}\right]$ con $t_{m}>0$.

(Es decir el intervalo de existencia de las soluciones depende de la dimensión de $V_{m}$ ). Para definir la solución en todo $[0, T]$ (extender el intervalo de solución) necesitamos obtener estimativas a priori para $u_{m}(t)$ y $v_{m}(t)$.

\section{$\underline{2^{a} \text { Etapa: Estimativas a Priori }}$}

$\underline{1^{\text {a }} \text { Estimativa: }}$

$$
\begin{gathered}
\left(u_{m}^{\prime \prime}(t), g_{j m}^{\prime}(t) \omega_{j}\right)+M\left(\left\|u_{m}\right\|(t)^{2}\right) a\left(u_{m}(t), g_{j m}^{\prime}(t) \omega_{j}\right)=\left(v_{m}^{2}(t) u_{m}(t), g_{j m}^{\prime}(t) \omega_{j}\right) ; j=1,2, \ldots, m \\
\left(u_{m}^{\prime \prime}(t), \sum_{j=1}^{m} g_{j m}^{\prime}(t) \omega_{j}\right)+M\left(u_{m}(t)^{2}\right) a\left(u_{m}(t), \sum_{j=1}^{m} g_{j m}^{\prime}(t) \omega_{j}\right)=\left(v_{m}^{2}(t) u_{m}(t), \sum_{j=1}^{m} g_{j m}^{\prime}(t) \omega_{j}\right) \\
\left(u_{m}^{\prime \prime}(t), u_{m}^{\prime}(t)\right)+M\left(u_{m}(t)^{2}\right) a\left(u_{m}(t), u_{m}^{\prime}(t)\right)=\left(v_{m}^{2}(t) u_{m}(t), u_{m}^{\prime}(t)\right)
\end{gathered}
$$

Usando las identidades:

$$
\begin{aligned}
& \left(u_{m}^{\prime \prime}(t), u_{m}^{\prime}(t)\right)=\frac{1}{2} \frac{d}{d t}\left|u_{m}^{\prime}(t)\right|^{2} \\
& a\left(u_{m}(t), u_{m}^{\prime}(t)\right)=\frac{1}{2} \frac{d}{d t}\left|u_{m}(t)\right|^{2}
\end{aligned}
$$


(Las cuales se verifican en el sentido de $\mathcal{D}^{\prime}\left(0, t_{m}\right)$

$$
\frac{1}{2} \frac{d}{d t}\left|u_{m}^{\prime}(t)\right|^{2}+\frac{1}{2} M\left(u_{m}(t)^{2}\right) \frac{d}{d t}\left\|u_{m}(t)\right\|^{2}=\left(v_{m}^{2}(t) u_{m}(t), u_{m}^{\prime}(t)\right)
$$

Análogamente:

$$
\frac{1}{2} \frac{d}{d t}\left|v_{m}^{\prime}(t)\right|^{2}+\frac{1}{2} M\left(v_{m}(t)^{2}\right) \frac{d}{d t}\left\|v_{m}(t)\right\|^{2}=\left(u_{m}^{2}(t) v_{m}(t), v_{m}^{\prime}(t)\right)
$$

Afirmación: $v_{m}^{2}(t) u_{m}(t), u_{m}^{2}(t) v_{m}(t) \in L^{2}(\Omega)$, es decir

$$
\left|v_{m}^{2}(t) u_{m}(t)\right|_{L^{2}(\Omega)} \leq C v_{m}(t)^{2}\left|u_{m}(t)\right|<\infty
$$

y

$$
\left|u_{m}^{2}(t) v_{m}(t)\right|_{L^{2}(\Omega)} \leq C u_{m}(t)^{2}\left|v_{m}(t)\right|<\infty
$$

Luego usando la desigualdad de Schwartz en (7) y (8),

$$
\begin{aligned}
& \frac{1}{2} \frac{d}{d t}\left|u_{m}^{\prime}(t)\right|^{2}+\frac{1}{2} M\left(u_{m}(t)^{2}\right) \frac{d}{d t}\left\|u_{m}(t)\right\|^{2} \leq\left(v_{m}^{2}(t) u_{m}(t), u_{m}^{\prime}(t)\right) \\
& \frac{1}{2} \frac{d}{d t}\left|v_{m}^{\prime}(t)\right|^{2}+\frac{1}{2} M\left(v_{m}(t)^{2}\right) \frac{d}{d t}\left\|v_{m}(t)\right\|^{2} \leq\left(u_{m}^{2}(t) v_{m}(t), v_{m}^{\prime}(t)\right)
\end{aligned}
$$

Ahora usando (9) y (10), luego hacemos

$$
\tilde{M}\left(\left\|u_{m}(t)\right\|^{2}\right)=\int_{0}^{\left\|u_{m}(t)\right\|^{2}} M(S) d S \text { y } \tilde{M}\left(\left\|v_{m}(t)\right\|^{2}\right)=\int_{0}^{\left\|v_{m}(t)\right\|^{2}} M(S) d S
$$

y observamos que:

$$
\frac{d}{d t} \tilde{M}\left(\left\|u_{m}(t)\right\|^{2}\right)=M\left(\left\|u_{m}(t)\right\|^{2}\right) \frac{d}{d t}\left\|u_{m}(t)\right\|^{2}
$$

Luego tendremos:

$$
\begin{aligned}
& \frac{1}{2} \frac{d}{d t}\left|u_{m}^{\prime}(t)\right|^{2}+\frac{d}{d t} \tilde{M}\left(\left\|u_{m}(t)\right\|^{2}\right) \leq C\left\|v_{m}(t)\right\|^{2}\left\|u_{m}(t)\right\| \cdot\left|u_{m}^{\prime}(t)\right| \\
& \frac{1}{2} \frac{d}{d t}\left|v_{m}^{\prime}(t)\right|^{2}+\frac{d}{d t} \tilde{M}\left(\left\|v_{m}(t)\right\|^{2}\right) \leq C\left\|u_{m}(t)\right\|^{2}\left\|v_{m}(t)\right\| \cdot\left|v_{m}^{\prime}(t)\right|
\end{aligned}
$$

Ahora sumando (12) y (13) tenemos:

$$
\begin{aligned}
& \frac{d}{d t}\left[\frac{1}{2}\left|u_{m}^{\prime}(t)\right|^{2}+\frac{1}{2}\left|v_{m}^{\prime}(t)\right|^{2}+\frac{1}{2} \tilde{M}\left(\left\|u_{m}(t)\right\|^{2}\right)+\frac{1}{2} \tilde{M}\left(\left\|v_{m}(t)\right\|^{2}\right)\right] \\
& \leq C\left\|v_{m}(t)\right\|^{2}\left|u_{m}(t)\right| \cdot\left|u_{m}^{\prime}(t)\right|+C\left\|u_{m}(t)\right\|^{2}\left|v_{m}(t)\right| \cdot\left|v_{m}^{\prime}(t)\right|
\end{aligned}
$$

A partir de ahora haremos uso de un tipo de estimativas inspiradas en las estimativas de Tartar [9].

Llamamos:

$$
\Psi_{m}(t)=\frac{1}{2}\left|u_{m}^{\prime}(t)\right|^{2}+\frac{1}{2}\left|v_{m}^{\prime}(t)\right|^{2}+\frac{1}{2} \tilde{M}\left(\left\|u_{m}(t)\right\|^{2}\right)+\frac{1}{2} \tilde{M}\left(\left\|v_{m}(t)\right\|^{2}\right)
$$

Observación: $M(S) \geq m_{0}>0$

Luego usamos (11) y Observación en (15)

$$
\Psi_{m}(t) \geq \frac{1}{2}\left|u_{m}^{\prime}(t)\right|^{2}+\frac{1}{2}\left|v_{m}^{\prime}(t)\right|^{2}+\frac{1}{2} m_{0}\left(\left\|u_{m}(t)\right\|^{2}\right)+\frac{1}{2} m_{0}\left(\left\|v_{m}(t)\right\|^{2}\right)
$$


Por tanto de (16) tenemos que:

$$
\left.\begin{array}{c}
\left\|u_{m}(t)\right\|^{2} \leq \frac{2}{m_{0}} \Psi_{m}(t) ;\left\|v_{m}(t)\right\|^{2} \leq \frac{2}{m_{0}} \Psi_{m}(t) \\
\left|u_{m}^{\prime}(t)\right| \leq \sqrt{2} \Psi_{m}{ }^{1 / 2}(t) ;\left|v_{m}^{\prime}(t)\right| \leq \sqrt{2} \Psi_{m}^{1 / 2}(t)
\end{array}\right\}
$$

Luego de (15) y (17) en (14) tenemos:

$$
\begin{aligned}
\Psi_{m}^{\prime}(t) & \leq C \frac{2}{m_{0}} \Psi_{m}(t) \frac{\sqrt{2}}{\sqrt{m_{0}}} \Psi_{m}^{1 / 2}(t) \sqrt{2} \Psi_{m}{ }^{1 / 2}(t) \\
++C \frac{2}{m_{0}} \Psi_{m}(t) \frac{\sqrt{2}}{\sqrt{m_{0}}} \Psi_{m}{ }^{1 / 2}(t) \sqrt{2} \Psi_{m}^{1 / 2}(t) & =\frac{4 C}{m_{0}{ }^{3 / 2}} \Psi_{m}{ }^{2}(t)+\frac{4 C}{m_{0}{ }^{3 / 2}} \Psi_{m}{ }^{2}(t)
\end{aligned}
$$

De donde:

$$
\Psi_{m}^{\prime}(t) \leq K \Psi_{m}^{2}(t)
$$

Con $K=\frac{8 C}{m_{0}{ }^{3 / 2}}>0$

De (19)tenemos

$$
\frac{d}{d t}\left[\Psi_{m}^{-1}(t)\right] \geq-K
$$

Integrando de 0 a $t$ tenemos:

$$
\Psi_{m}^{-1}(t) \geq \Psi_{m}^{-1}(0)-K t
$$

Observamos que:

Luego:

$$
\Psi_{m}{ }^{-1}(0)-K t>0 \text { si y solo si } t<\frac{\Psi_{m}^{-1}(0)}{K}
$$

$$
\Psi_{m}(t) \leq \frac{1}{\Psi_{m}{ }^{-1}(0)-K t} \text { para } \mathrm{t} \in\left[0, \frac{\Psi_{m}{ }^{-1}(0)}{K}\right]
$$

Sea:

$$
\Psi_{m}(0) \leq \frac{1}{2}\left|u_{1}\right|^{2}+\frac{1}{2}\left|v_{1}\right|^{2}+\frac{1}{2} \tilde{M}\left(\left\|u_{0}\right\|^{2}\right) \frac{1}{2} \tilde{M}\left(\left\|v_{0}\right\|^{2}\right)+1
$$

Para $m \geq r_{0}$ suficientemente grande

Afirmación:

$$
\Psi_{m}(t) \leq 2 A ; \forall t \in\left[0, \frac{A^{-1}}{2 K}\right] \subset\left[0, \frac{\Psi_{m}^{-1}(0)}{K}\right]
$$

Por tanto de (15) y (22) tenemos que:

$$
\begin{aligned}
& \left(u_{m}\right) \text { y }\left(v_{m}\right) \text { es acotado en } L^{\infty}\left(0, T_{0} ; H_{0}^{1}(\Omega)\right) \\
& \left(u_{m}^{\prime}\right) \text { y }\left(v_{m}^{\prime}\right) \text { es acotado en } L^{\infty}\left(0, T_{0} ; L^{2}(\Omega)\right)
\end{aligned}
$$

\section{$\underline{2^{a} \text { Estimativa: }}$}

Multiplicando en (1) y (2) por $\lambda_{j} g_{j m}^{\prime}(t)$ y $\lambda_{j} h_{j m}^{\prime}(t)$ respectivamente y luego sumando de $j=1,2, \ldots, m$ tenemos:

$$
\begin{aligned}
& \left(u_{m}^{\prime \prime}(t), \sum_{j=1}^{m} g_{j m}^{\prime}(t) \lambda_{j} \omega_{j}\right) \\
& +M\left(\left\|u_{m}(t)\right\|^{2}\right) a\left(u_{m}(t), \sum_{j=1}^{m} g_{j m}^{\prime}(t) \lambda_{j} \omega_{j}\right)=\left(v_{m}^{2}(t) u_{m}(t), \sum_{j=1}^{m} g_{j m}^{\prime}(t) \lambda_{j} \omega_{j}\right)
\end{aligned}
$$


Ahora usamos el hecho de que $-\Delta \omega_{j}=\lambda_{j} \omega_{j}$ en (23) se tiene:

$$
\begin{gathered}
\left(u_{m}^{\prime \prime}(t),-\Delta \sum_{j=1}^{m} g_{j m}^{\prime}(t) \omega_{j}\right)+M\left(\left\|u_{m}(t)\right\|^{2}\right) a\left(u_{m}(t),-\Delta \sum_{j=1}^{m} g_{j m}^{\prime}(t) \omega_{j}\right) \\
=\left(v_{m}^{2}(t) u_{m}(t),-\Delta \sum_{j=1}^{m} g_{j m}^{\prime}(t) \omega_{j}\right)
\end{gathered}
$$

Luego:

$$
\left(u_{m}^{\prime \prime}(t),-\Delta u_{m}^{\prime}(t)\right)+M\left(\left\|u_{m}(t)\right\|^{2}\right) a\left(u_{m}(t),-\Delta u_{m}^{\prime}(t)\right)=\left(v_{m}^{2}(t) u_{m}(t),-\Delta u_{m}^{\prime}(t)\right)
$$

Ahora hacemos uso de las siguientes identidades:

$$
\left.\begin{array}{l}
\left(u_{m}^{\prime \prime}(t),-\Delta u_{m}^{\prime}(t)\right)=\frac{1}{2} \frac{d}{d t}\left|\nabla u_{m}^{\prime}(t)\right|^{2} \\
a\left(u_{m}(t),-\Delta u_{m}^{\prime}(t)\right)=\frac{1}{2} \frac{d}{d t}\left|\Delta u_{m}(t)\right|^{2}
\end{array}\right\} \text { en el sentido de las distribuciones }
$$

Entonces

$$
\frac{1}{2} \frac{d}{d t}\left|\nabla u_{m}^{\prime}(t)\right|^{2}+M\left(\left\|u_{m}(t)\right\|^{2}\right) \frac{1}{2} \frac{d}{d t}\left|\Delta u_{m}(t)\right|^{2}=\left(v_{m}^{2}(t) u_{m}(t),-\Delta u_{m}^{\prime}(t)\right)
$$

Por el teorema de Green en el $2^{\circ}$ miembro de (24); y como $\left\|u_{m}(t)\right\|^{2} \leq K$ (por la $1^{a}$ estimativa) Y $M \in C^{1}\left(\left[0,+\infty[)\right.\right.$ entonces $m_{0}=\underset{0 \leq\left\|u_{m}(t)\right\|^{2} \leq K}{\operatorname{Min} . M}\left(\left\|u_{m}(t)\right\|^{2}\right)$

Luego tenemos:

$$
\frac{1}{2} \frac{d}{d t}\left|\nabla u_{m}^{\prime}(t)\right|^{2}+\frac{1}{2} m_{0} \frac{d}{d t}\left\|\Delta u_{m}(t)\right\|^{2} \leq\left(\nabla\left(v_{m}^{2}(t) u_{m}(t)\right), \nabla u_{m}^{\prime}(t)\right)
$$

Por la desigualdad de Schwartz tenemos:

$$
\frac{1}{2} \frac{d}{d t}\left|\nabla u_{m}^{\prime}(t)\right|^{2}+\frac{1}{2} m_{0} \frac{d}{d t}\left\|\Delta u_{m}(t)\right\|^{2} \leq\left|\nabla\left(v_{m}^{2}(t) u_{m}(t)\right)\right| \cdot\left|\nabla u_{m}^{\prime}(t)\right|
$$

Análogamente:

$$
\frac{1}{2} \frac{d}{d t}\left|\nabla v_{m}^{\prime}(t)\right|^{2}+\frac{1}{2} m_{0} \frac{d}{d t}\left\|\Delta v_{m}(t)\right\|^{2} \leq\left|\nabla\left(u_{m}^{2}(t) v_{m}(t)\right)\right| \cdot\left|\nabla v_{m}^{\prime}(t)\right|
$$

Sumando miembro a miembro (25) y (26)

$$
\begin{gathered}
\frac{d}{d t}\left[\frac{1}{2}\left|\nabla u_{m}^{\prime}(t)\right|^{2}+\frac{1}{2}\left|\nabla v_{m}^{\prime}(t)\right|^{2}+\frac{m_{0}}{2}\left|\Delta u_{m}(t)\right|^{2}+\frac{m_{0}}{2}\left|\Delta v_{m}(t)\right|^{2}\right] \leq \\
\quad \leq\left|\nabla\left(v_{m}^{2}(t) u_{m}(t)\right)\right| \cdot\left|\nabla u_{m}^{\prime}(t)\right|+\left|\nabla\left(u_{m}^{2}(t) v_{m}(t)\right)\right| \cdot\left|\nabla v_{m}^{\prime}(t)\right|
\end{gathered}
$$

Tenemos que:

$$
\begin{aligned}
& \nabla\left(v_{m}^{2}(t) u_{m}(t)\right)=\nabla\left(v_{m}^{2}(t)\right) u_{m}(t)+v_{m}^{2}(t) \cdot \nabla u_{m}(t) \\
&=2 v_{m}(t) u_{m}(t) \nabla v_{m}(t)+v_{m}^{2}(t) \cdot \nabla u_{m}(t) \\
&\left|\nabla\left(v_{m}^{2}(t) u_{m}(t)\right)\right| \leq\left|2 v_{m}(t) u_{m}(t) \nabla v_{m}(t)\right|+\left|v_{m}^{2}(t) \cdot \nabla u_{m}(t)\right| \\
&=2\left|v_{m}(t)\right| \cdot\left|u_{m}(t)\right| \cdot\left|\nabla v_{m}(t)\right|+\left|v_{m}^{2}(t)\right| \cdot\left|\nabla u_{m}(t)\right| \\
& \leq 2 C_{1}\left\|v_{m}(t)\right\| \cdot\left\|u_{m}(t)\right\| \cdot\left|\nabla v_{m}(t)\right|+C_{2}\left\|v_{m}(t)\right\| \cdot\left\|v_{m}(t)\right\| \cdot\left|\nabla u_{m}(t)\right|
\end{aligned}
$$

Pues: $H_{0}^{1}(t) \rightarrow L^{2}$ es una inmersión continúa 
Retomemos $(*)$

$$
\begin{gathered}
\leq 2 C_{3}\left\|v_{m}(t)\right\|_{H^{2}}\left\|u_{m}(t)\right\|_{H^{2}}\left|\nabla v_{m}(t)\right|+C_{4}\left\|v_{m}(t)\right\|_{H^{2}}\left\|v_{m}(t)\right\|_{H^{2}}\left|\nabla u_{m}(t)\right| \\
=C_{5}\left|\Delta v_{m}(t)\right| \cdot\left|\Delta u_{m}(t)\right| \cdot\left|\nabla v_{m}(t)\right|+C_{6}\left|\Delta v_{m}(t)\right|^{2}\left|\nabla u_{m}(t)\right|
\end{gathered}
$$

Así tenemos:

$$
\left|\nabla\left(v_{m}^{2}(t) u_{m}(t)\right)\right| \leq C_{5}\left|\Delta v_{m}(t)\right| \cdot\left|\Delta u_{m}(t)\right| \cdot\left|\nabla v_{m}(t)\right|+C_{6}\left|\Delta v_{m}(t)\right|^{2}\left|\nabla u_{m}(t)\right|
$$

Con $C_{5}, C_{6}>0$

Análogamente:

$$
\left|\nabla\left(u_{m}^{2}(t) v_{m}(t)\right)\right| \leq C_{7}\left|\Delta v_{m}(t)\right| \cdot\left|\Delta u_{m}(t)\right| \cdot\left|\nabla u_{m}(t)\right|+C_{8}\left|\Delta u_{m}(t)\right|^{2}\left|\nabla v_{m}(t)\right|
$$

Con $C_{7}, C_{8}>0$

Reemplazando (28) y (29) en (27) tenemos:

$$
\begin{aligned}
& \frac{d}{d t}\left[\frac{1}{2}\left|\nabla u_{m}^{\prime}(t)\right|^{2}+\frac{1}{2}\left|\nabla v_{m}^{\prime}(t)\right|^{2}+\frac{m_{0}}{2}\left|\Delta u_{m}(t)\right|^{2}+\frac{m_{0}}{2}\left|\Delta v_{m}(t)\right|^{2}\right] \leq \\
\leq & \left(C_{5}\left|\Delta v_{m}(t)\right| \cdot\left|\Delta u_{m}(t)\right| \cdot\left|\nabla v_{m}(t)\right|+C_{6}\left|\Delta v_{m}(t)\right|^{2}\left|\nabla u_{m}(t)\right|\right)\left|\nabla u_{m}^{\prime}(t)\right| \\
+ & \left(C_{7}\left|\Delta v_{m}(t)\right| \cdot\left|\Delta u_{m}(t)\right| \cdot\left|\nabla u_{m}(t)\right|+C_{8}\left|\Delta u_{m}(t)\right|^{2}\left|\nabla v_{m}(t)\right|\right)\left|\nabla v_{m}^{\prime}(t)\right|
\end{aligned}
$$

Ahora empleamos la técnica de Tartar para lograr nuestra acotación:

Llamamos:

$$
\Psi_{m}(t)=\frac{1}{2}\left|\nabla u_{m}^{\prime}(t)\right|^{2}+\frac{1}{2}\left|\nabla v_{m}^{\prime}(t)\right|^{2}+\frac{m_{0}}{2}\left|\Delta u_{m}(t)\right|^{2}+\frac{m_{0}}{2}\left|\Delta v_{m}(t)\right|^{2}
$$

Desarrollando y usando la $1^{a}$ estimativa se tiene:

$$
\Psi_{m}^{\prime}(t) \leq K \Psi_{m}^{3 / 2}(t)
$$

Luego: $\Psi_{m}^{3 / 2}(t) \Psi_{m}^{\prime}(t) \leq K$ es decir: $\frac{d}{d t}\left[\Psi_{m}^{-1 / 2}(t)\right] \geq-\frac{1}{2} K$

Integrando de 0 a $t$, entonces

$$
\Psi_{m}^{-1 / 2}(t) \leq \frac{1}{\Psi_{m}^{-1 / 2}(0)-K t} \text { para } t \in\left[0, \frac{\Psi_{m}^{-1 / 2}(0)}{K}\right]
$$

Se sabe que:

$$
\Psi_{m}(0) \leq A=\frac{1}{2}\left|\nabla u_{1}\right|^{2}+\frac{1}{2}\left|\nabla v_{1}\right|^{2}+\frac{m_{0}}{2}\left|\Delta u_{0}\right|^{2}+\frac{m_{0}}{2}\left|\Delta v_{0}\right|^{2}+1
$$

Para $m \geq r_{0}$ suficientemente grande

Afirmación:

$$
\Psi_{m}^{\prime}(t) \leq \sqrt{2} A ; \forall t \in\left[0, \frac{A^{-1}}{2 K}\right] \subset\left[0, \frac{\Psi_{m}^{-1}(0)}{K}\right]
$$

Por tanto de (31)y la afirmación tenemos que:

$$
\frac{1}{2}\left|\nabla u_{m}^{\prime}(t)\right|^{2}+\frac{1}{2}\left|\nabla v_{m}^{\prime}(t)\right|^{2}+\frac{m_{0}}{2}\left|\Delta u_{m}(t)\right|^{2}+\frac{m_{0}}{2}\left|\Delta v_{m}(t)\right|^{2} \leq C ; t \in\left[0, T_{2}\right]
$$

de este modo

$$
\frac{1}{2}\left|\nabla u_{m}^{\prime}(t)\right|^{2}+\frac{1}{2}\left|\nabla v_{m}^{\prime}(t)\right|^{2}+m_{0}\left|\Delta u_{m}(t)\right|^{2}+m_{0}\left|\Delta v_{m}(t)\right|^{2} \leq C_{1} ; t \in\left[0, T_{2}\right]
$$


Donde $C_{1}=2 C=2 \sqrt{2} A$

$\underline{3^{a} \text { Estimativa: }}$

$$
\begin{aligned}
& \left(u_{m}^{\prime \prime}(t), u_{m}^{\prime \prime}(t)\right)=M\left(u_{m}(t)^{2}\right)\left(\Delta u_{m}(t), u_{m}^{\prime \prime}(t)\right)+\left(v_{m}^{2}(t) u_{m}(t), u_{m}^{\prime \prime}(t)\right) \\
& \left(v_{m}^{\prime \prime}(t), v_{m}^{\prime \prime}(t)\right)=M\left(v_{m}(t)^{2}\right)\left(\Delta v_{m}(t), v_{m}^{\prime \prime}(t)\right)+\left(u_{m}^{2}(t) v_{m}(t), v_{m}^{\prime \prime}(t)\right)
\end{aligned}
$$

(36) equivale a:

$$
\begin{aligned}
\left|u_{m}^{\prime \prime}(t)\right|^{2} & \leq M\left(u_{m}(t)^{2}\right)\left|\Delta u_{m}(t)\right|\left|u_{m}^{\prime \prime}(t)\right|+\left|v_{m}^{2}(t) u_{m}(t)\right|\left|u_{m}^{\prime \prime}(t)\right| \\
& \uparrow \\
& \text { Aplicando la desigualdad de Schwartz }
\end{aligned}
$$

Usamos el resultado de que:

$$
\left|v_{m}^{2}(t) u_{m}(t)\right| \leq C\left\|v_{m}(t)\right\|^{2}\left\|u_{m}(t)\right\| ; \text { donde } C>0
$$

Luego (37) implica:

$$
\left|u_{m}^{\prime \prime}(t)\right| \leq M\left(\left\|u_{m}(t)\right\|^{2}\right)\left|\Delta u_{m}(t)\right|+C\left\|v_{m}(t)\right\|^{2}\left\|u_{m}(t)\right\|
$$

En forma similar

$$
\left|v_{m}^{\prime \prime}(t)\right| \leq M\left(\left\|v_{m}(t)\right\|^{2}\right)\left|\Delta v_{m}(t)\right|+C\left\|u_{m}(t)\right\|^{2}\left\|v_{m}(t)\right\|
$$

Por la $1^{a}$ estimativa y la pertenencia de $M$, entonces:

$$
M\left(\left\|u_{m}(t)\right\|^{2}\right) \leq \underset{0 \leq\|u(t)\|^{2} \leq C}{\operatorname{Max} . M}\left(\left\|u_{m}(t)\right\|^{2}\right)=C_{0}
$$

Por la $2^{a}$ estimativa (33) que $\left\|\Delta u_{m}(t)\right\|^{2} \leq C_{1}$

Entonces de (38) tenemos:

$$
\left|u_{m}^{\prime \prime}(t)\right| \leq C_{0} C_{1}^{1 / 2}+C C^{1 / 2}=\text { constante, } \forall t \in\left[0, T_{0}\right] \text { con } T_{0}=\min \left\{T_{1}, T_{2}\right\}
$$

Análogamente:

$$
\left|u_{m}^{\prime \prime}(t)\right| \leq \text { constante } ; \forall t \in\left[0, T_{0}\right]
$$

Luego:

$$
\left|u_{m}^{\prime \prime}(t)\right|+\left|v_{m}^{\prime \prime}(t)\right| \leq K, K>0
$$

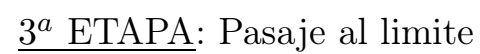

Las ecuaciones aproximadas son:

$$
\begin{gathered}
\left(u_{m}^{\prime \prime}(t), \omega_{j}\right)+M\left(\left\|u_{m}(t)\right\|^{2}\right) a\left(u_{m}(t), \omega_{j}\right)=\left(v_{m}^{2}(t) u_{m}(t), \omega_{j}\right) \\
\left(v_{m}^{\prime \prime}(t), \omega_{j}\right)+M\left(\left\|v_{m}(t)\right\|^{2}\right) a\left(v(t), \omega_{j}\right)=\left(u_{m}^{2}(t) v_{m}(t), \omega_{j}\right) ; \omega_{j} \in V_{m}, j=1,2, \ldots, m
\end{gathered}
$$

De las estimativas $1^{a}$ y de la $2^{a}$, y considerando $\omega_{j} \in H_{0}^{1}(\Omega) \cap H^{2}(\Omega), j=1,2, \ldots, m$ Luego tomando supremo esencial tenemos:

$$
\underset{t \in] 0, T_{0}[}{S u p} . e s s\left\|u_{m}(t)\right\|_{H_{0}^{t}(\Omega) \cap H^{2}(\Omega)} \leq \sqrt{C_{4}}
$$

Esto equivale:

$$
\left\|u_{m}\right\|_{L^{\infty}\left(0, T_{0}, H_{0}^{1}(\Omega) \cap H^{2}(\Omega)\right)} \leq \sqrt{C_{4}}
$$


es decir: $\left(u_{m}\right)$ es acotada en

$$
L^{\infty}\left(0, T_{0}, H_{0}^{1}(\Omega) \cap H^{2}(\Omega)\right)
$$

Análogamente $\left(v_{m}\right)$ es acotada en

$$
\left.L^{\infty}\left(0, T_{0}, H_{0}^{1}(\Omega) \cap H^{2}(\Omega)\right)\right)
$$

También de (35) se tiene que: $\left|\nabla u_{m}^{\prime}(t)\right|_{L^{2}(\Omega)}^{2} \leq C_{1} ; \forall t \in\left[0, T_{0}\right]$

Esto equivale a:

$$
\left|u_{m}^{\prime}(t)\right|_{H_{0}^{1}(\Omega)}^{2} \leq C_{1} ; \forall t \in\left[0, T_{0}\right]
$$

Tomando supremo esencial: $\left\|u_{m}^{\prime}\right\|_{L^{\infty}\left(0, T_{0}, H_{0}^{1}(\Omega)\right)} \leq \sqrt{C_{1}}$

es decir: $\left(u_{m}^{\prime}\right)$ es acotada en

$$
L^{\infty}\left(0, T_{0}, H_{0}^{1}(\Omega)\right)
$$

análogamente $\left(v_{m}^{\prime}\right)$ es acotada en

$$
L^{\infty}\left(0, T_{0}, H_{0}^{1}(\Omega)\right)
$$

De la $3^{a}$ estimativa y luego tomando supremo esencial en $] 0, T_{0}[$ tenemos:

$$
\left\|u_{m}^{\prime \prime}\right\|_{L^{\infty}\left(0, T_{0}, L^{2}(\Omega)\right)} \leq \text { constante }
$$

es decir: $\left(u_{m}^{\prime \prime}\right)$ es acotada en

$$
L^{\infty}\left(0, T_{0}, L^{2}(\Omega)\right)
$$

Análogamente $\left(v_{m}^{\prime \prime}\right)$ es acotada en

$$
L^{\infty}\left(0, T_{0}, L^{2}(\Omega)\right)
$$

Por otro lado de (43) y por el teorema de Alaoglu- Bourbaki se tiene que existe una subsucesión de $\left(u_{m}^{\prime \prime}\right)$ y $y \in L^{\infty}\left(0, T_{0}, L^{2}(\Omega)\right)$ tal que:

$$
u_{m}^{\prime \prime} \stackrel{*}{\rightarrow} Y \text { débil*en } L^{\infty}\left(0, T_{0}, L^{2}(\Omega)\right)
$$

esto es: $u_{m}^{\prime \prime} \rightarrow Y$ débil * en $L^{\infty}\left(0, T_{0}, L^{2}(\Omega)\right)$

Afirmamos que $u^{\prime \prime}=Y$.

Considerando que:

$$
u_{m}^{\prime} \stackrel{*}{\rightarrow} u^{\prime} \text { débil*en } L^{\infty}\left(0, T_{0}, L^{2}(\Omega)\right)
$$

Se tiene que:

$$
\left\langle u_{m}^{\prime}, \omega\right\rangle \rightarrow\left\langle u^{\prime}, \omega\right\rangle, \quad \forall \omega \in L^{1}\left(0, T_{0}, L^{2}(\Omega)\right)
$$

Donde hay que observar que $L^{1}\left(0, T_{0}, L^{2}(\Omega)\right)^{\prime}=L^{\infty}\left(0, T_{0}, L^{2}(\Omega)\right)$

La notación de (44) significa que:

$$
\int_{0}^{T_{0}}\left(u_{m}^{\prime}(t), \omega(t)\right) d t \rightarrow \int_{0}^{T_{0}}\left(u^{\prime}(t), \omega(t)\right) d t, \quad \forall \omega \in L^{1}\left(0, T_{0} ; L^{2}(\Omega)\right)
$$

Notemos que el producto interno en $L^{2}(\Omega):\left(u_{m}^{\prime}(t), \omega(t)\right)$ tiene sentido.

Luego tiene sentido el producto en $L^{2}(\Omega):\left(u_{m}^{\prime}(t), \omega(t)\right)$, del mismo modo tiene sentido $\left(u^{\prime}(t), \omega(t)\right)$.

Continuando a partir de (45), tenemos que para $\omega(t)=v \theta(t)$ con

$$
v \in H_{0}^{1}(\Omega) \rightarrow L^{2}(\Omega) \text { y } \theta \in \mathcal{D}\left(0, T_{0}\right) \rightarrow L^{2}\left(0, T_{0}\right)
$$

se tiene que

$$
\omega \in L^{1}\left(0, T_{0}, L^{2}(\Omega)\right) \text { y } \omega(t) \in L^{2}(\Omega)
$$


luego para este $\omega(t)=v \theta(t)$ en (45) se tiene:

$$
\int_{0}^{T_{0}}\left(u_{m}^{\prime}(t), v\right) \theta(t) d t \rightarrow \int_{0}^{T_{0}}\left(u^{\prime}(t), v\right) \theta(t) d t, \quad \forall v \in H_{0}^{1}(\Omega) \text { y } \forall \theta \in \mathcal{D}\left(0, T_{0}\right)
$$

de este modo

$$
\left\langle\left(u_{m}^{\prime}(t), v\right), \theta\right\rangle \rightarrow\left\langle\left(u^{\prime}(t), v\right), \theta\right\rangle, \forall v \in H_{0}^{1}(\Omega) \text { y } \forall \theta \in \mathcal{D}\left(0, T_{0}\right)
$$

es decir:

$$
\left(u_{m}^{\prime}(t), v\right) \rightarrow\left(u^{\prime}(t), v\right), \forall v \in H_{0}^{1}(\Omega)
$$

en el sentido de $\mathcal{D}^{\prime}\left(0, T_{0}\right)$ (Es decir en el sentido de las distribuciones).

Luego derivando en el sentido de las distribuciones tenemos:

$$
\begin{gathered}
\frac{d}{d t}\left(u_{m}^{\prime}(t), v\right) \rightarrow \frac{d}{d t}\left(u^{\prime}(t), v\right), \forall v \in H_{0}^{1}(\Omega) e n \mathcal{D}^{\prime}\left(0, T_{0}\right) \\
\left\langle\frac{d}{d t}\left(u_{m}^{\prime}(t), v\right), \varphi\right\rangle \rightarrow\left\langle\frac{d}{d t}\left(u^{\prime}(t), v\right), \varphi\right\rangle, \forall v \in H_{0}^{1}(\Omega) y \forall \varphi \in \mathcal{D}\left(0, T_{0}\right)
\end{gathered}
$$

Esto equivale a:

$$
-\int_{0}^{T_{0}}\left(u_{m}^{\prime}(t), v\right) \varphi^{\prime}(t) d t \rightarrow-\int_{0}^{T_{0}}\left(u^{\prime}(t), v\right) \varphi^{\prime}(t) d t
$$

Integrando por partes, luego equivale en particular para $\omega_{j} \in H_{0}^{1}(\Omega)$ tenemos:

$$
\left\langle\left(u_{m}^{\prime \prime}(t), \omega_{j}\right), \varphi\right\rangle \rightarrow\left\langle\left(u^{\prime \prime}(t), \omega_{j}\right), \varphi\right\rangle, \forall j=1,2, \ldots \varphi \in \mathcal{D}\left(0, T_{0}\right)
$$

Lo cual equivale a decir:

$$
\left(u_{m}^{\prime \prime}(t), \omega_{j}\right) \rightarrow\left(u^{\prime \prime}(t), \omega_{j}\right), \quad \forall j=1,2, \ldots \quad \mathcal{D}^{\prime}\left(0, T_{0}\right)
$$

Análogamente: siguiendo los mismos pasos, se sigue que

$$
\left(v_{m}^{\prime \prime}(t), \omega_{j}\right) \rightarrow\left(v^{\prime \prime}(t), \omega_{j}\right), \quad \forall j=1,2, \ldots \quad \mathcal{D}^{\prime}\left(0, T_{0}\right)
$$

De la $1^{a}$ Estimativa, entonces por el teorema de Alaoglu - Bourbaki existe una subsucesión de $\left(u_{m}\right)$ tal que:

$$
u_{m} \stackrel{*}{\rightarrow} u \text { débil*en } L^{\infty}\left(0, T_{0}, H_{0}^{1}(\Omega)\right)
$$

De ello tenemos que:

$$
u_{m} \rightarrow u \text { débil en } L^{2}\left(0, T_{0}, H_{0}^{1}(\Omega)\right)
$$

Luego de (48) por la definición de convergencia débil tenemos que:

$$
\left\langle\omega, u_{m}\right\rangle \rightarrow\langle\omega, u\rangle, \forall \omega \in L^{2}\left(0, T_{0}, H_{0}^{1}(\Omega)\right)
$$

Continuando a partir de (49);

(49) significa (por definición de dicha convergencia en (48) que:

$$
\int_{0}^{T_{0}}\left\langle\omega(t), u_{m}^{\prime}(t)\right\rangle_{H_{0}^{1}(\Omega) x H_{0}^{1}(\Omega)} d t \rightarrow \int_{0}^{T_{0}}\langle\omega(t), u(t)\rangle_{H_{0}^{1}(\Omega) x H_{0}^{1}(\Omega)} d t
$$

Como $\omega(t), u_{m}(t) \in H_{0}^{1}(\Omega)$ la dualidad de $H^{-1}(\Omega)$ con $H_{0}^{1}(\Omega)$ se convierte en un producto interno en $H_{0}^{1}(\Omega)$ que es un espacio de Hilbert real, luego el producto interno es simétrico, así de $(50)$ se tiene:

$$
\int_{0}^{T_{0}}\left(\left(u_{m}(t), \omega(t)\right)\right) d t \rightarrow \int_{0}^{T_{0}}(u(t), \omega(t)) d t
$$

Donde: 
$(()$,$) denota el producto interno en H_{0}^{1}(\Omega)$ denotado también por $a(u, v)=((u, v))$. Luego (51) se escribe como:

$$
\int_{0}^{T_{0}} a\left(u_{m}(t), \omega(t)\right) d t \rightarrow \int_{0}^{T_{0}} a(u(t), \omega(t)) d t, \quad \forall \omega \in L^{2}\left(0, T_{0}, H_{0}^{1}(\Omega)\right)
$$

Luego se sigue, lo cual equivale a decir:

$$
a\left(u_{m}(t), \omega_{j}\right) \rightarrow a\left(u(t), \omega_{j}\right), \quad \forall j=1,2, \ldots \underbrace{e n \mathcal{D}^{\prime}\left(0, T_{0}\right)}_{\begin{array}{c}
\text { Es decir en el sentido de } \\
\text { las distribuciones sobre }] 0, T_{0} \text { [ }
\end{array}}
$$

Análogamente siguiendo los mismos pasos, se sigue:

$$
a\left(v_{m}(t), \omega_{j}\right) \rightarrow a\left(v(t), \omega_{j}\right), \forall j=1,2, \ldots \text { en } \mathcal{D}^{\prime}\left(0, T_{0}\right)
$$

Afirmamos: $M\left(\left|\nabla u_{m}(t)\right|^{2}\right) \rightarrow M\left(|\nabla u(t)|^{2}\right)$ fuerte en $L^{2}\left(0, T_{0}\right)$

Luego, haciendo cálculos, equivale a decir:

$$
M\left(\left\|u_{m}(t)\right\|^{2}\right) a\left(u_{m}(t), \omega_{j}\right) \rightarrow M\left(\|u(t)\|^{2}\right) a\left(u(t), \omega_{j}\right), \forall j=1,2, \ldots \text { en } \mathcal{D}^{\prime}\left(0, T_{0}\right)
$$

Análogamente:

$$
M\left(\left\|v_{m}(t)\right\|^{2}\right) a\left(v_{m}(t), \omega_{j}\right) \rightarrow M\left(\|v(t)\|^{2}\right) a\left(v(t), \omega_{j}\right), \forall j=1,2, \ldots \text { en } \mathcal{D}^{\prime}\left(0, T_{0}\right)
$$

$4^{a}$ Etapa: Convergencia de los términos no lineales

No olvidemos que estamos considerando $\Omega$ abierto de $\mathbb{R}^{n} ; n=1,2$

En este caso se obtiene que

$$
\left|v_{m}^{2}(t) u_{m}(t)\right|_{L^{2}(\Omega)} \leq C\left|u_{m}(t) v_{m}(t)\right|_{L^{2}(\Omega)} \cdot\left\|v_{m}(t)\right\| \leq C_{1}
$$

Análogamente

$$
\left|u_{m}^{2}(t) v_{m}(t)\right|_{L^{2}(\Omega)} \leq C\left|u_{m}(t) v_{m}(t)\right|_{L^{2}(\Omega)} \cdot\left\|u_{m}(t)\right\| \leq C_{2}
$$

Para (56) y (57) estamos haciendo uso de un trabajo de Medeiros - Milla Miranda [5] (página $176)$.

Luego de (56) tenemos usando supremos esencial que:

$$
\left(v_{m}^{2} u_{m}\right) \text { es acotado en } L^{\infty}\left(0, T_{0}, L^{2}(\Omega)\right)
$$

Del mismo modo se tiene:

$$
\left(u_{m}^{2} v_{m}\right) \text { es acotado en } L^{\infty}\left(0, T_{0}, L^{2}(\Omega)\right)
$$

Ahora, la siguiente etapa es estudiar la convergencia en casi todo punto de los términos no lineales $v_{m}^{2} u_{m}$ y $u_{m}^{2} v_{m}$ para cada $t \in\left[0, T_{0}\right]$

De la primera estimativa y de (42), luego se sigue que: $u_{m}$ y $v_{m}$ están acotadas en $H^{\prime}\left(Q_{0}\right)$, pues con inmersión compactada, luego existen subsucesiones $\left(u_{m}\right)$ y $\left(v_{m}\right)$ tales que:

$u_{m} \rightarrow u$ y $v_{m} \rightarrow v$ fuerte en $L^{2}\left(Q_{0}\right)$, luego se sigue que

$$
u_{m} \rightarrow u \text { y } v_{m} \rightarrow v \text { en casi todo punto de } Q_{0}
$$

Luego se tiene que:

$$
u_{m}^{2} \rightarrow u^{2} \text { y } v_{m}^{2} \rightarrow v^{2} \text { en casi todo punto de } Q_{0}
$$

Combinando (60) y (61) se tiene:

$$
v_{m}^{2} u_{m} \rightarrow v^{2} u \text { en casi todo punto en } Q_{0}
$$




$$
u_{m}^{2} v_{m} \rightarrow u^{2} v \text { en casi todo punto en } Q_{0}
$$

De (62), (59), (62), (63) y el Lema de Lions, esto es equivalente a:

$$
\int_{0}^{T_{0}}\left\langle v_{m}^{2}(t) u_{m}(t), \varphi(t)\right\rangle_{L^{P} x L^{Q}} d t \rightarrow \int_{0}^{T_{0}}\left\langle v^{2}(t) u(t), \varphi(t)\right\rangle_{L^{P} \times L^{Q}} d t
$$

En particular para $p=2$, siguiendo, equivale a

$$
\left(v_{m}^{2}(t) u_{m}(t), \omega_{j}\right) \rightarrow\left(v^{2}(t) u(t), \omega_{j}\right), \forall j=1,2, \ldots \text { en } \mathcal{D}^{\prime}\left(0, T_{0}\right)
$$

Análogamente

$$
\left(u_{m}^{2}(t) v_{m}(t), \omega_{j}\right) \rightarrow\left(u^{2}(t) v(t), \omega_{j}\right), \forall j=1,2, \ldots \text { en } \mathcal{D}^{\prime}\left(0, T_{0}\right)
$$

Ahora de (46), (54),(64) y haciendo $m \rightarrow+\infty$

Análogamente: De (47), (55),(65) y haciendo $m \rightarrow+\infty$

Luego ello prueba que existe $(u, v)$ solución del sistema:

$$
\begin{aligned}
& u^{\prime \prime}-M\left(\|u(t)\|^{2}\right) \Delta u=v^{2} u \\
& v^{\prime \prime}-M\left(\|v(t)\|^{2}\right) \Delta v=u^{2} v
\end{aligned}
$$

en el sentido débil.

\section{Conclusiones}

El sistema $(*)$ es una generalización del caso que considera $M \equiv 1$ que es el caso típico de un sistema acoplado de Klein - Gordon, al respecto se puede ver el trabajo de Medeiros - Milla Miranda [5] quienes estudian un sistema con acoplamiento más general de la forma

$$
|v|^{p+2}|\mu|^{p} \mu \quad \text { y } \quad|\mu|^{p+2}|v|^{p} v
$$




\section{REFERENCIAS BIBLIOGRÁFICAS}

[1] Brezis,H. (1983). Analyse Fonctionelle, Theorie et Applications, Massor, Paris.

[2] Carrillo, D. L. (1988). Nao Existencia de Solucoes fracas Globais de una Equacao Hiperbolica. Atas de 28 S.B.A., Rio de Janeiro.

[3] Kirchhoff, G. (1985). Vorlesungerk Uber Mechanic Tauberk, Leipzig.

[4] Lions, J.L. (1969). Quelques Mathodes de Resolution des problems aux limites nonlineares, Donod Paris.

[5] Medeiros, L.A., Milla Miranda M. (1986). Weak Solutions for a System of non Linear Klein Gordon Equations. Annali di Matematica Pura ed Applicata (IV). Vol CXLVI, 173-183.

[6] Milla Miranda M., Medeiros, L.A. (1987). On the existence of global Solutions of a coupled nonlinear Klein Gordon Equations. Funkcialaj Ekvadoj. Vol 30, 147-161.

[7] Perla M. (1995). On a Mixed Problem for a class of non linear Klein Gordon Equations. 21 Seminario Brasileiro.

[8] Schwartz, L. (1950). Theorie des Distributions. Tome I, II. Hermann Ed. Paris.

[9] Tartar, L. (1978). Topics in Nonlinear analysis. Publications Mathematiques D'orsay. 